\title{
Dielectric Property Studies of Biologically Compatible Brushite Single Crystals Used as Bone Graft Substitute
}

\author{
M. P. Binitha, P. P. Pradyumnan*
}

Department of Physics, University of Calicut, Kerala, India.

Email: *drpradyumnan@gmail.com

Received January $13^{\text {th }}, 2013$; revised February $26^{\text {th }}, 2013$; accepted March $29^{\text {th }}, 2013$

Copyright (C) 2013 M. P. Binitha, P. P. Pradyumnan. This is an open access article distributed under the Creative Commons Attribution License, which permits unrestricted use, distribution, and reproduction in any medium, provided the original work is properly cited.

\begin{abstract}
The electrical characterization of bone is essential for the better understanding of the role of electrical stimulation in bone remodeling. Calcium Hydrogen Phosphate Dihydrate or brushite $\left(\mathrm{CaHPO}_{4} 2 \mathrm{H}_{2} \mathrm{O}\right)$ has been used in bone substitution owing to their fast resorption under physiological condition. Brushite is a suitable matrix for osteoconductive bone grafts. In this work, Calcium Hydrogen Phosphate single crystals have been grown by single diffusion gel growth technique. The powder XRD studies revealed the monoclinic structure of the grown crystals. The vibrational analysis of the crystals is done with FTIR spectroscopy and the major functional groups and their assigned vibrations are discussed. The frequency dependence of dielectric constant and ac conductivity at different temperatures have been studied in detail. This study shows decrease in the dielectric constant with the increase in frequency and temperature. The variation of ac conductivity is found to be increasing with frequency and decreasing with temperature.
\end{abstract}

Keywords: Bone Grafts; Brushite; Gel Growth; Dielectric Properties; AC Conductivity

\section{Introduction}

Bone is one of the natural materials made up of organic as well as inorganic components. Although bone has the ability to repair itself following damage, in the case of complex bone fractures which fails to heal properly, causing a significant health risk to the patients, bone grafting is needed. Bone grafts may be autograft, allograft or synthetic with similar mechanical properties to bone. Most bone grafts are expected to be reabsorbed and replaced as the natural bone heals over a few months time. The ideal bone substitute should have osteoconductive and osteoinductive properties [1].

Approximately $60 \%$ of the bone graft substitutes currently available involve ceramics, either alone or in combination with another material. These include calcium sulfate, bioactive glass, and calcium phosphate [1]. In the past 20 years the most frequently used synthetic bone graft has been the calcium orthophosphates ceramics. One class of calcium orthophosphates used in bone grafting applications are the calcium phosphate cements, which are the combination of a solid component containing one or more calcium orthophosphate powder with an aqueous

"Corresponding author. solution [2]. The calcium phosphate cements can overcome many problems associated with autologous grafting such as scarcity of grafting material and secondary mordibility and the risk of biological contamination associated with allograft [3]. In addition, calcium phosphates are osteoconductive, osteointegrative and in some cases, osteoinductive. Depending on the $\mathrm{pH}$ value of the cement paste the end product of the cement setting reaction may be either brushite or hydroxyapatite. The advantage of hydroxyapatite over brushite is that it is usually set at a $\mathrm{pH}$ value closer to the physiological environment. Hence it has been more frequently used. However, brushite is more soluble than hydroxyapatite in physiological condition and hence is more completely resorbed after its implantation in animal bodies [2]. Thus brushite is a suitable matrix as osteoconductive bone grafts $[4,5]$. Several clinical studies have shown that injectable brushite cements are capable of regenerating oral and maxillofacial bone in atrophic areas, buccal dehiscence defects and maxillary sinuses. Also brushite cements have been used to fill bone defects created by pathological tumors [6]. In the newly formed natural bone itself, the occurrence of brushite is reported [7].

While electrical stimulation is used for healing frac- 
tures, the electrical characterization of bone has much clinical importance. Furthermore, earlier studies have suggested that electrical and dielectric properties of natural bone depend on the bone density and could, therefore, be used to predict bone strength. Information on the inter relationship between electrical and dielectric properties with bone mineral density would enable the estimation of field distribution during electrical stimulation. In this context the electrical characterization of the bone grafting material is worthwhile and hence we have investigated the electric as well as dielectric properties of brushite. The electrical measurements were conducted in a wide frequency range, as motivated by the fact that electromagnetic pulses used in the stimulation of bone healing contain a wide range of frequencies as well [8].

\section{Experimental}

The growth of calcium phosphate has been carried out using single diffusion of calcium chloride through the hydrosilica gel medium. Because of the viscous nature of gel, this growth technique provides an in vitro model for crystallization of biomolecules [9]. The silica gel was set by acidification of sodium meta silicate solution of specific gravity 1.03 with $0.5 \mathrm{M}$ orthophosphoric acid to get a pH of $6[10,11]$. This gel is found to set after 24 hours. After assuring proper gel setting, $7 \mathrm{ml}$ of $0.5 \mathrm{M}$ calcium chloride solution was slowly poured over it, without disturbing the gel surface. Small crystals appeared in the gel, which were mostly platelets or needle shaped. After a growth period of 6 weeks the crystals were harvested and characterization studies were carried out. The structural analyses were done by X-ray diffraction method using $\mathrm{Cu}-\mathrm{K} \alpha$ monochromator of wavelength $1.541 \AA$ (XRD). The vibrational analysis was done by Fourier Transform Infra Red (FTIR) spectroscopy on the powdered sample by the standard $\mathrm{KBr}$ pellet method in the region 400 $4000 \mathrm{~cm}^{-1}$. Differential Scanning Calorimeter (DSC) was used to measure the thermal characteristics of grown crystals. Dielectric studies were conducted by LCR Hi TESTER 3532-50.

\section{Result and Discussion}

XRD pattern of the grown crystal is given in Figure 1. XRD studies confirmed that the crystal structure is that of brushite having monoclinic structure. The values of cell parameters are $\mathrm{a}=5.812 \AA, \mathrm{b}=15.18 \AA, \mathrm{c}=6.239 \AA$, $\alpha=90^{\circ}, \beta=116.43^{\circ}, \gamma=90.00^{\circ}$ (JCPDS 72-0713).

FTIR spectrum of brushite crystals is shown in Figure 2. The broad absorption peak between $3500-2400 \mathrm{~cm}^{-1}$ is due to $\mathrm{O}-\mathrm{H}$ stretching vibration. The $\mathrm{H}-\mathrm{O}-\mathrm{H}$ bending gives rise to absorption at $1648.27 \mathrm{~cm}^{-1}$. The absorptions at 1214.35 and $1132.03 \mathrm{~cm}^{-1}$ are due to $\mathrm{P}=\mathrm{O}$ associated stretching vibrations. The absorption at $1065.32 \mathrm{~cm}^{-1}$ is

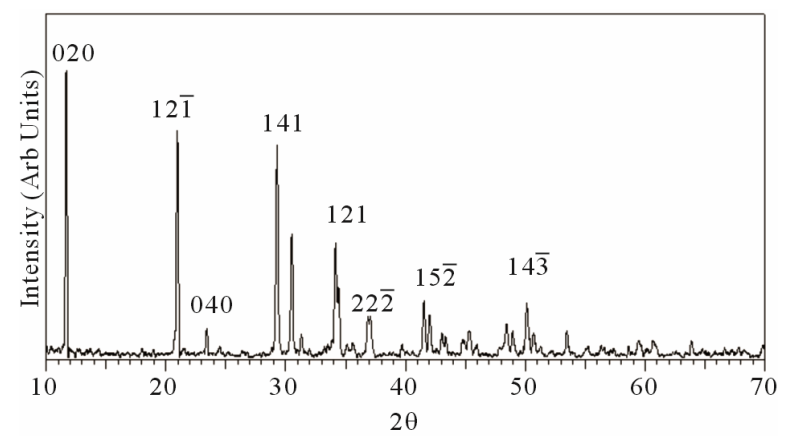

Figure 1. XRD pattern of grown brushite.

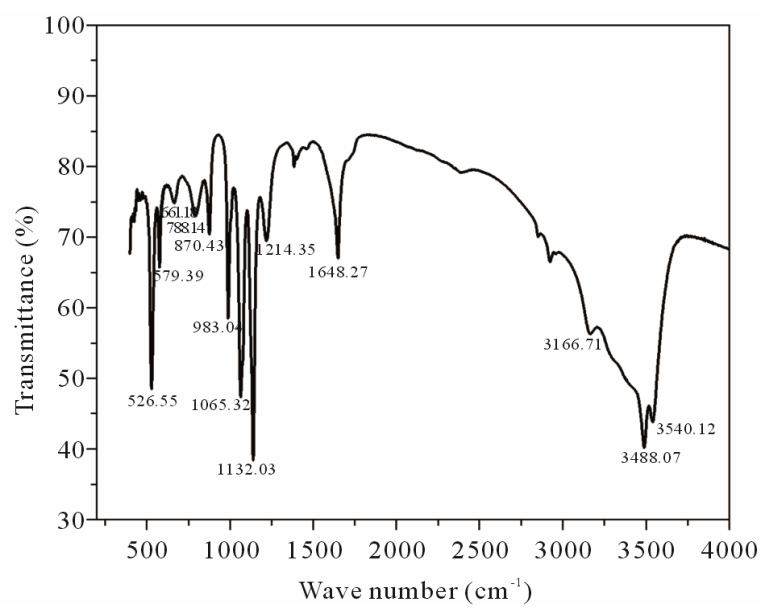

Figure 2. FT-IR spectrum of grown brushite.

due to $\mathrm{P}=\mathrm{O}$ stretching vibrations. The $\mathrm{P}-\mathrm{O}-\mathrm{P}$ asymmetric stretching vibrations give rise to absorptions at 983.04, 870.43 and $788.14 \mathrm{~cm}^{-1}$. The absorptions at 661.13 , $579.39,526.55 \mathrm{~cm}^{-1}$ are due to (H-O-) $\mathrm{P}=\mathrm{O}$ for acid phosphates $[12,13]$.

The differential thermal analysis was done with a heat flow from $50.00^{\circ} \mathrm{C}$ to $375.00^{\circ} \mathrm{C}$ at $10.00^{\circ} \mathrm{C} / \mathrm{min}$ using the instrument Perkin Elmer DSC 4000. The thermograms of brushite crystals (Figure 3) show two endothermic peaks, a broad one at around $149.38^{\circ} \mathrm{C}$ corresponding to the onset of evaporation of water of crystallisation and therefore the partial dehydration of brushite and a well defined peak at $190.85^{\circ} \mathrm{C}$ associated with the phase transition of the crystal due to the total dehydration [13]. At this temparature brushite is completely transformed to monatite according to the relation

$$
\mathrm{CaHPO}_{4} 2 \mathrm{H}_{2} \mathrm{O} \rightarrow \mathrm{CaHPO}_{4}+2 \mathrm{H}_{2} \mathrm{O}
$$

\section{Conductivity Studies}

The variation of dielectric constant $\left(\varepsilon_{\mathrm{r}}\right)$ and ac conductivity $\left(\sigma_{\mathrm{ac}}\right)$ of the crystal with frequency of applied field ranging from $100 \mathrm{~Hz}$ to $5 \mathrm{MHz}$ is studied at different temperatures. The samples were finely ground and made in the form of pellets. The dielectric constant $\left(\varepsilon_{\mathrm{r}}\right)$ is 
calculated using the relation

$$
\varepsilon_{\mathrm{r}}=\frac{\mathrm{Cd}}{\varepsilon_{0} \mathrm{~A}}
$$

and the ac conductivity is calculated by the relation

$$
\sigma_{\mathrm{ac}}=\varepsilon_{0} \varepsilon_{\mathrm{r}} \omega \tan \delta
$$

where $\mathrm{C}$ is the capacitance, $\mathrm{d}$ is the thickness, $\mathrm{A}$ is the area of cross section of pellet and $\tan \delta$ is the dielectric relaxation of the sample. It is observed that the dielectric constant and ac conductivity decrease with increasing temperature as shown in Figures $\mathbf{4}$ and $\mathbf{5}$ respectively. Also the values of dielectric constant decreases with increasing frequency, while the ac conductivity value increases with increasing frequency. The dielectric constant is high at lower frequency region and decreases with increasing frequencies. The high value of dielectric constant at low frequencies is attributed to space charge polarization. At lower range of frequencies dielectric constant decreases drastically with frequency becoming a constant at higher frequencies. The polarization occurs due to the local displacement of electrons, which is the effect of the electronic exchange of the number of ions in the crystal. As frequency increases the electron exchange cannot follow the electric field and the polarization becomes independent of frequency.

Variation of dielectric constant with temperature is generally attributed to the orientational polarization, crystal expansion, the presence of impurities and crystal defects. When temperature increases the dielectric constant decreases, as evident from the Figure 4. The thermal energy disrupts the ion dipole interaction which is responsible for polarization at higher temperatures, causing the relaxation of polarization. The ac conductivity increases with frequency and decreases with temperature [14,15].

During open surgery for bone grafting or total hip replacement, there is no method for direct measurement of

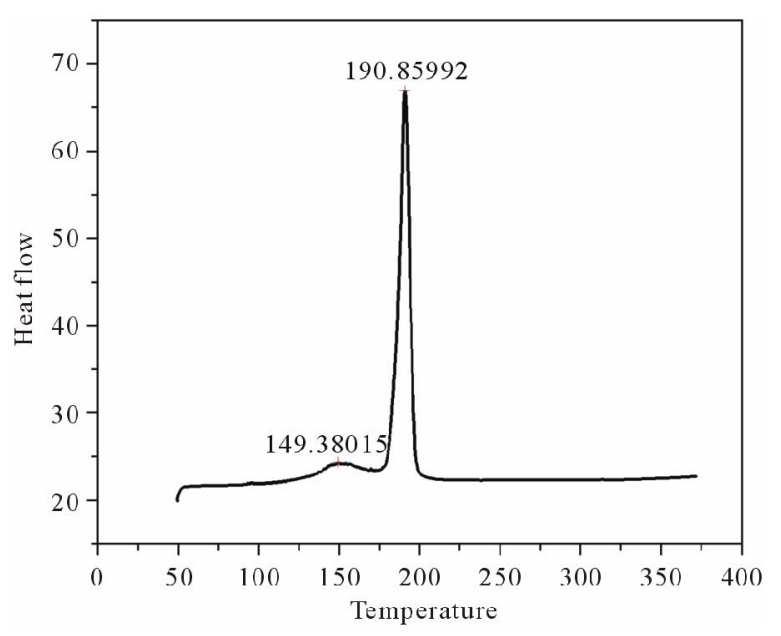

Figure 3. DSC plot of grown brushite.

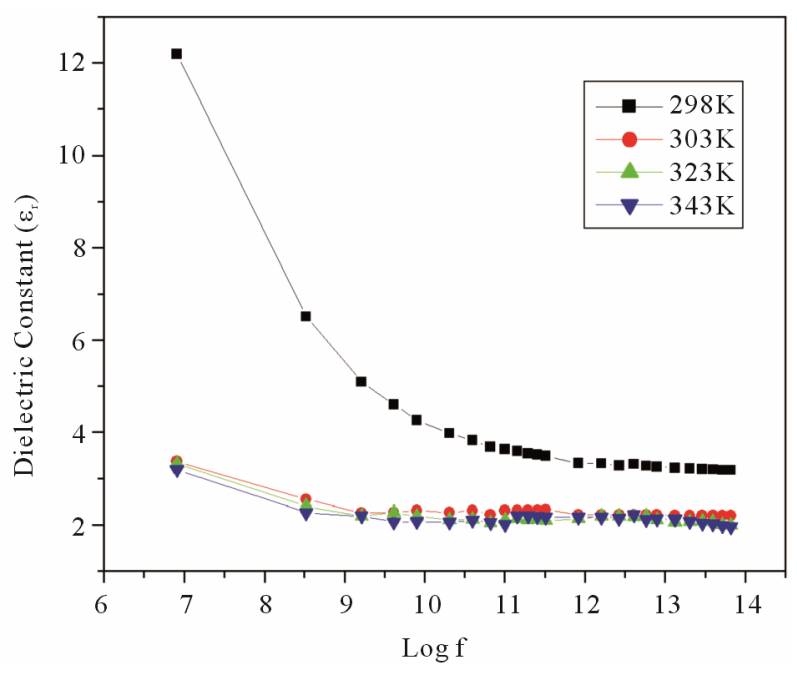

Figure 4. Variation of dielectric constant with frequency.

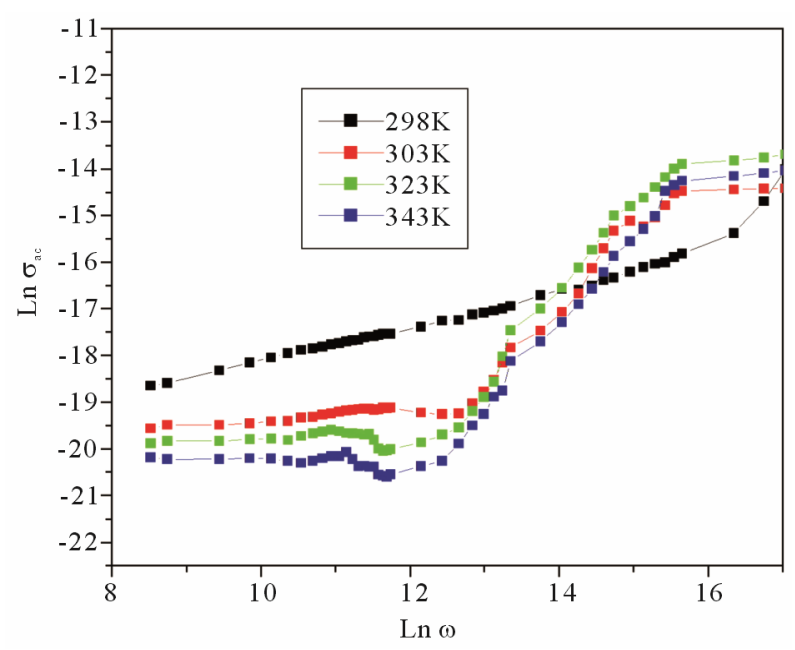

Figure 5. Variation of ac conductivity with frequency.

bone quality. Electrical measurements utilizing openended probe technology are potentially feasible in this kind of application. In addition, better understanding of the relationships between bone density, mechanical, electrical and dielectric properties may help us to improve techniques related to electrical stimulation and follow-up of healing processes in fractured bone. Several studies were made to study the variation of dielectric constant of bone as a function of frequency and found that the dielectric constant decreases with frequency $[16,17]$.

\section{Conclusion}

Brushite crystals are grown successfully by gel method and crystal structure is determined to be monoclinic. The FTIR studies confirmed the major functional groups in the crystal. The dielectric constant shows decrease with frequency, attaining a constant value at higher frequencies. Also the dielectric constant decreases with tempera- 
ture. The ac conductivity is found to be increasing with frequency and decreasing with temperature.

\section{Acknowledgements}

M.P.B would like to acknowledge UGC, Govt. of India, for the award of FDP (KLCA 062 TF 01). She is also thankful to University of Calicut and Govt. College, Kodanchery, for their support.

\section{REFERENCES}

[1] W. R. Moore, S. E. Graves and G. I. Bain, "Synthetic Bone Graft Substitutes," ANZ Journal of Surgery, Vol. 71, No. 6, 2001, pp. 354-361.

[2] Z. D. Xia, L. M. Grover, Y. Z. Huang, I. E. Adamopoulos, U. Gbureck, J. T. Triffitt, R. M. Shelton and J. E. Barralet, "In Vitro Biodegradation of Three Brushite Calcium Phosphate Cements by a Macrophage Cell-Line," Biomaterials, Vol. 27, No. 26, 2006, pp. 5457-4565.

doi:10.1016/j.biomaterials.2006.04.030

[3] G. Penel, N. Leroy, P. Van Landuyt, B. Flautre, P. Hardouin, J. lemaitre and G. Leroy, "Raman Microspectrometry Studies of Brushite Cement: In Vivo Evolution in a Sheep Model," Bone, Vol. 25, No. 2, 1999, pp. 81S-84S. doi:10.1016/S8756-3282(99)00139-8

[4] H. N. Lim, A. Kassim, N. M. Huang, M. A. Armo, P. S. Khiew and W. S. Chiu, "Preparation and Characterization of Brushite Crystals Using High Internal Phase Emulsion," Colloid Journal, Vol. 71, No. 6, 2009, pp. 793-802. doi:10.1134/S1061933X09060088

[5] S. V. Dorozhkin, "Calcium Orthophosphates as Bioceramics: State of the Art," Journal of Functional Biomaterials, Vol. 1, No. 1, 2010, pp. 22-107

[6] F. Tamimi, Z. Sheikh and J. Barralet, "Dicalcium Phosphate Cements: Brushite and Monetite," Acta Biomaterialia, Vol. 8, No. 2, 2012, pp. 474-487. doi:10.1016/j.actbio.2011.08.005

[7] W. F. Neuman, M. W. Neuman, A. G. Diamond, J. Menanteau and W. Gibbons, "Blood: Bone Disequilibrium. VI. Studies of the Solubility Characteristics of Brushite: Apatite Mixtures and Their Stabilization by Noncollagenous Proteins of Bone," Calcified Tissue International, Vol. 34, No. 1, 1982, pp. 149-157

[8] J. Sierpowska, J. Toyras, M. A. Hakulinen, S. Saarakkala, J. S. Jurvelin and R. Lappalainen, "Electrical and Dielectric Properties of Bovine Trabecular Bone-Relationships with Mechanical Properties and Mineral Density," Physics in Medicine and Biology, Vol. 48, No. 6, 2003, pp. 775-786. doi:10.1088/0031-9155/48/6/306

[9] K. Suguna and C. Sekar, "Role of Strontium on the Crystallization of Calcium Hydrogen Phosphate Dihydrate (CHPD)," Journal of Minerals and Materials Characterization and Engineering, Vol. 10, No. 7, 2011, pp. 625636. doi: $10.1002 /$ crat.200610826

[10] C. Justin Raj, G. Mangalam, S. Mary Navis Priya, J. Mary Linet, C. Vesta, S. Dinakaran, B. Milton Boaz and S. Jerome Das, "Growth and Characterization of Nonlinear Optical Zinc Hydrogen Phosphate Single Crystal Grown in Silica Gel," Crystal Research and Technology, Vol. 42, No. 4, 2007, pp. 344-348. doi: $10.1002 /$ crat.200610826

[11] V. Mahalakshmi, A. Lincy, J. Thomas and K. V. Saban, "Crystal Growth and Characterization of a New Co-Ordination Complex-Barium Tetrakismaleate Dihydrate," Journal of Physics and Chemistry of Solids, Vol. 73, No. 4, 2012, pp. 584-588. doi:10.1016/j.jpcs.2011.12.012

[12] G. Madhurambal, R. Subha and S. C. Mojumdar, "Crystallizaion and Thermal Characterization of Calcium Hydrogen Phosphate Dihydrate Crystals," Journal of Thermal Analysis and Calorimetry, Vol. 96, No. 1, 2009, pp. 73-76. doi:10.1007/s10973-008-9841-1

[13] K Rajendran and C. Dale Keefe, "Growth and Characterization of Calcium Hydrogen Phosphate Dihydrate Crystals from Single Diffusion Gel Technique," Crystal Research and Technology, Vol. 45, No. 9, 2010, pp. 939945. doi:10.1002/crat.200900700

[14] B. Parekh, M. Joshi and A. Vaidya, "Characterisation and Inhibitive Study of Gel Grown Hydroxyapatite Crystals at Physiological Temperature," Journal of Crystal Growth, Vol. 310, No. 7, 2008, pp. 1749-1753. doi:10.1016/j.jcrysgro.2007.11.219

[15] K. Arora, V. Patel, B. Amin and A. Kothari, "Dielectric Behaviour of Strontium Tartrate Single Crystals," Bulletin of Material Science, Vol. 27, No. 2, 2004, pp. 141-147. doi:10.1007/BF02708496

[16] G. N. Reddy and S. Saha, "Electrical and Dielectric Properties of Wet Bone as a Function of Frequency," IEEE Transactions on Biomedical Engineering, Vol. BME-31, No. 3, 1984, pp. 296-303.

[17] A. A. Narino, R. O. Becker and C. H. Bachman, "Dielectric Determination of Bound Water of Bone," Physics in Medicine and Biology, Vol. 12, No. 3, 1967, pp. 367-378. doi:10.1088/0031-9155/12/3/309 